\title{
Dermatology Research and Therapy
}

CASE REPORT

\section{Linear Iga Bullous Dermatosis in Crohn's Disease: A Case Report}

\section{Ruba Ibrahim, MD, Gil Armoni, MD, Georgina-Maria Sarika, MD and Vered Molho-Pessach, MD*}

Department of Dermatology, Hebrew University of Jerusalem, Israel

*Corresponding author: Vered Molho-Pessach, MD, Department of Dermatology, Hadassah-Hebrew University Medical Center, Jerusalem, Israel

\begin{abstract}
Linear IgA bullous dermatosis (LABD) is a rare autoimmune, vesiculo-bullous skin disease. It has been previously reported in association with inflammatory bowel disease, particularly ulcerative colitis. We report a case of LABD in a patient suffering from Crohn's disease who received tumor necrosis factor- $\alpha$ inhibitor therapy and developed LABD several months later.
\end{abstract}

\section{Keywords}

Crohn's disease, Linear IgA bullous dermatosis, Infliximab

\section{Introduction}

Linear IgA bullous dermatosis (LABD) is a rare autoimmune, vesiculobullous skin disease, characterized by linear deposition of IgA at the dermo-epidermal junction resulting in subepidermal tense bullae and neutrophilic infiltration. Clinical manifestation can vary from erythematous, urticarial, annular plaques to vesicular or bullous lesions arranged in a herpetiform pattern, described as "cluster of jewels" sign. It has been reported in association with malignancies, infections, connective tissue diseases, medications, and inflammatory bowel disease, particularly ulcerative colitis [1]. Drug induced LABD is most commonly triggered by vancomycin. Other medications that have been reported as a potential trigger include penicillins, cephalosporins and nonsteroidal anti-inflammatory agents [2].

We report a patient with Crohn's disease patient who developed LABD several months following tumor necrosis factor- $\alpha$ inhibitor therapy with infliximab. So far, only 4 cases of LABD have been associated with Crohn's disease.

\section{Case Report}

A 40-year-old patient was diagnosed with Crohn's disease in 2017. He was treated with prednisone and adalimumab with minimal improvement. In March 2018, he started treatment with Infliximab. Eight months later, he developed a pruritic widespread vesiculo-bullous rash. On examination the patient was noted to have over his extremities, abdomen, back, and buttocks numerous vesicles arranged in a rosette pattern, as well as erythematous edematous annular plaques. Vesicles and erosions were also noted in the soft palate (Figure 1).

A $3 \mathrm{~mm}$ punch biopsy from the abdomen showed

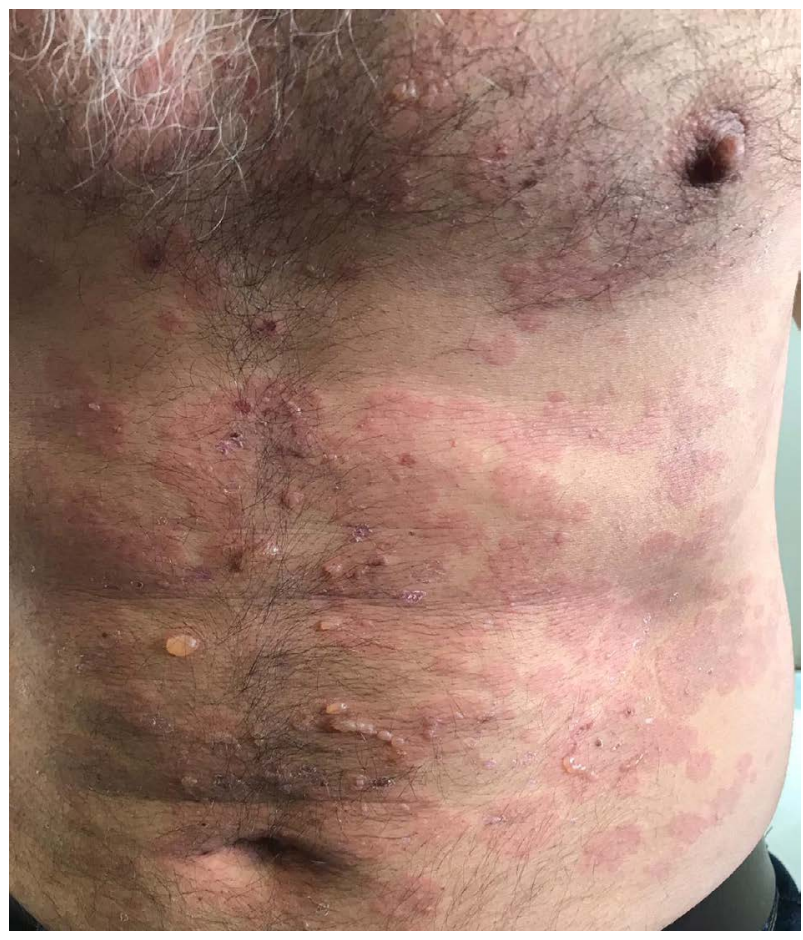

Figure 1: Erythematous edematous annular plaques as well as numerous vesicles arranged in a rosette pattern. 
sub-epidermal blistering and neutrophilic infiltration in the papillary dermis. Direct immunoflourescence showed linear deposition of IgA along the basement membrane zone (BMZ). Indirect immunofluorescence (IIF) showed circulating IgA BMZ antibodies in a titer of 1:80.

Based on the clinical and pathological findings the patient was diagnosed with linear IgA bullous dermatosis (LABD).

Due to the possibility of drug related LABD, and due to inadequate response of his Crohn's disease to the infliximab, the latter was discontinued in consultation with his gastroenterologist. The patient experienced a flare-up of Crohn's disease that necessitated systemic corticosteroids. At the same time, no improvement was observed in the vesicular rash and treatment with topical and systemic corticosteroids was initiated.

Avlosulfone is considered first line treatment in LABD, but due to glucose 6 phosphatase dehydrogenase deficiency in our patient, other treatment options were considered [1]. Azathioprine was given but due to increased levels of diastase several weeks following initiation of treatment and suspected drug induced pancreatitis, it was discontinued. Doxycycline $100 \mathrm{mg}$ twice daily was started, resulting in only short-term improvement. Meanwhile, ustekinumab was given for the Crohn's disease with good response, but no improvement was observed in the vesiculobullous rash.

Alternative treatment options for LABD were considered, such as rituximab, mycophenolate mofetil and colchicine. In order to avoid prolonged and heavy immunosuppression, colchicine was suggested, but the patient was not interested. He remained free of treatment for a few months with gradual and spontaneous improvement of his LABD.

\section{Discussion}

So far, only 4 cases of LABD in association with Crohn's disease have been published. Three of the cases were reported in adult patients with a diagnosis of LABD that appeared during the onset of Crohn's disease [3-5]. The fourth case was a 13-year-old girl who was diagnosed with Crohn's disease 6 months prior to onset of the LABD [6].

In our case, the bowel disease preceded the onset of LABD, and persisted even after bowel disease was well controlled with ustekinumab.

There are very few case reports on the association of infliximab and LABD. Two cases reported infliximab treatment for ulcerative colitis (UC) which was followed by an outbreak of LABD $[7,8]$. On the other hand, another case of LABD was reported in a patient with UC who was successfully treated with infliximab [9].

Up to date, 34 cases of LABD associated with UC have been reported. In most of the cases, there was no association between LABD and activity of UC, and in some patients, persistent LABD was reported even after control of UC was achieved [10].

It is unclear how inflammatory bowel disease affects the pathogenesis and course of LABD. But it is believed that immunoglobulin activation may be induced by inflammatory bowel disease, triggering IgA cross-reactivity against antigens in the epidermis, particularly in the lamina lucida and sublamina densa regions of the basement membrane, causing an increase in IgA production, leading to the development of LABD [1].

In the patient presented here, it remains unclear whether the etiology of LABD is medication (infliximab) induced or associated with his Crohn's disease.

This case highlights the fact that LABD can develop as a result of the presence of systemic inflammatory disease or secondary to medications, and indicates that a deep investigation of a patient's background is required in LABD.

\section{Conflict of Interest}

The authors have no conflicts of interest to disclose.

\section{Funding}

None.

\section{Authors Contributions}

RI wrote the manuscript; GA, GMS, and VMP participated in analysis and discussion of the data, in addition to proofreading of the manuscript. All authors approve the current version of the manuscript for publication.

\section{References}

1. Genovese G, Venegoni L, Fanoni D, Muratori S, Berti E, et al. (2019) Linear IgA bullous dermatosis in adults and children: A clinical and immunopathological study of 38 patients. Orphanet Journal of Rare Diseases 14: 115.

2. Jha P, Swanson K, Stromich J, Michalski BM, Olasz E (2017) A rare case of vancomycin-induced linear immunoglobulin A bullous dermatosis. Case Reports in Dermatological Medicine.

3. Torres T, Sanches M, Selores M (2010) Linear IgA bullous disease in a patient with Crohn's disease. Acta Dermatoven APA 19: 29-31.

4. Barberis $C$, Doutre MS, Bioulac-Sage $P$, Pompougnac $E$, Beylot C (1988) Linear IgA bullous dermatosis associated with Crohn's disease. Gastroenterologie Clinique et Biologique 12: 76-77.

5. Birnie AJ, Perkins W (2005) A case of linear IgA disease occurring in a patient with colonic Crohn's disease. British Journal of Dermatology 153: 1050-1052.

6. Nanda A, Dvorak R, Al-Sabah H, Madda JP, Anim JT (2006) Association of linear $\lg A$ bullous disease of childhood with Crohn's disease. International Journal of Dermatology 45: 1184-1186.

7. Bryant KD, DeNunzio MJ, Ford MJ (2016) Linear IgA dermatosis after infliximab infusion for ulcerative colitis. JAAD Case Reports 2: 448-450. 
8. Hoffmann J, Hadaschik E, Enk A, Stremmel W, Gauss A (2015) Linear IgA bullous dermatosis secondary to infliximab therapy in a patient with ulcerative colitis. Dermatology 231: 112-115.

9. Yamada S, Makino T, Jinnin M, Sakai K, Fukushima S, et al. (2013) Association of linear IgA bullous disease with ul- cerative colitis: a case of successful treatment with infliximab. Dermatology 227: 295-298.

10. Sonoyama H, Mishima $\mathrm{Y}$, Ishihara S, Oshima N, Moriyama I, et al. (2019) Ten-year follow-up study of linear immunoglobulin A dermatosis complicated with ulcerative colitis. Clinical Journal of Gastroenterology 13: 164-169. 